Louisiana State University

LSU Digital Commons

Faculty Publications

Department of Biological Sciences

$11-20-2014$

\title{
The drivers of tropical speciation
}

\author{
Brian Tilston Smith \\ Louisiana State University \\ John E. McCormack \\ American Museum of Natural History \\ Andrés M. Cuervo \\ Louisiana State University \\ Michael J. Hickerson \\ City College of New York \\ Alexandre Aleixo \\ Museu Paraense Emilio Goeldi
}

See next page for additional authors

Follow this and additional works at: https://digitalcommons.Isu.edu/biosci_pubs

\section{Recommended Citation}

Smith, B., McCormack, J., Cuervo, A., Hickerson, M., Aleixo, A., Cadena, C., Pérez-Emán, J., Burney, C., Xie, X., Harvey, M., Faircloth, B., Glenn, T., Derryberry, E., Prejean, J., Fields, S., \& Brumfield, R. (2014). The drivers of tropical speciation. Nature, 515 (7527), 406-409. https://doi.org/10.1038/nature13687

This Article is brought to you for free and open access by the Department of Biological Sciences at LSU Digital Commons. It has been accepted for inclusion in Faculty Publications by an authorized administrator of LSU Digital Commons. For more information, please contact ir@lsu.edu. 


\section{Authors}

Brian Tilston Smith, John E. McCormack, Andrés M. Cuervo, Michael J. Hickerson, Alexandre Aleixo, Carlos Daniel Cadena, Jorge Pérez-Emán, Curtis W. Burney, Xiaoou Xie, Michael G. Harvey, Brant C.

Faircloth, Travis C. Glenn, Elizabeth P. Derryberry, Jesse Prejean, Samantha Fields, and Robb T. Brumfield 


\title{
The drivers of tropical speciation
}

\author{
Brian Tilston Smith ${ }^{1,2 *}$, John E. McCormack ${ }^{1} \uparrow$, Andrés M. Cuervo ${ }^{1,3} \uparrow$, Michael. J. Hickerson ${ }^{4,5}$, Alexandre Aleixo ${ }^{6}$, \\ Carlos Daniel Cadena ${ }^{7}$, Jorge Pérez-Emán ${ }^{8,9}$, Curtis W. Burney ${ }^{1,3} \dagger$, Xiaoou Xie ${ }^{4}$, Michael G. Harvey ${ }^{1,3}$, Brant C. Faircloth ${ }^{10} \dagger$, \\ Travis C. Glenn ${ }^{11}$, Elizabeth P. Derryberry ${ }^{1}$, Jesse Prejean $^{1,3}$, Samantha Fields ${ }^{1,3}$ \& Robb T. Brumfield ${ }^{1,3 *}$
}

Since the recognition that allopatric speciation can be induced by large-scale reconfigurations of the landscape that isolate formerly continuous populations, such as the separation of continents by plate tectonics, the uplift of mountains or the formation of large rivers, landscape change has been viewed as a primary driver of biological diversification. This process is referred to in biogeography as vicariance ${ }^{1}$. In the most species-rich region of the world, the Neotropics, the sundering of populations associated with the Andean uplift is ascribed this principal role in speciation ${ }^{2-5}$. An alternative model posits that rather than being directly linked to landscape change, allopatric speciation is initiated to a greater extent by dispersal events, with the principal drivers of speciation being organism-specific abilities to persist and disperse in the landscape $e^{6,7}$. Landscape change is not a necessity for speciation in this model $^{8}$. Here we show that spatial and temporal patterns of genetic differentiation in Neotropical birds are highly discordant across lineages and are not reconcilable with a model linking speciation solely to landscape change. Instead, the strongest predictors of speciation are the amount of time a lineage has persisted in the landscape and the ability of birds to move through the landscape matrix. These results, augmented by the observation that most species-level diversity originated after episodes of major Andean uplift in the Neogene period, suggest that dispersal and differentiation on a matrix previously shaped by large-scale landscape events was a major driver of avian speciation in lowland Neotropical rainforests.

In the species-rich Neotropics, the origins of biodiversity are usually linked to changes to the Earth's landscape over geological time ${ }^{2-5,9,10}$. Palaeogeographic studies indicate that Andean mountain building during the Neogene catalysed tumultuous changes in the lowlands, including formation of the Amazon River system, closure of the Isthmus of Panama, and the isolation of humid lowland forests east and west of the Andes by montane habitats and the aridification of the Caribbean lowlands in northern South America ${ }^{4}$. These large-scale landscape changes are hypothesized to have driven speciation by fragmenting species distributions that were formerly continuous, a process that can generate congruent spatial and temporal patterns of genetic differentiation in co-distributed lineages, especially for lineages with similar ecological characteristics. Bolstering support for the importance of landscape change driving isolation in this region, time-calibrated phylogenies of a taxonomically diverse group of organisms encompassing a broad range of ecologies and dispersal abilities indicate that many modern Neotropical lineages originated during time periods associated with major reconfigurations of the landscape, presumably signifying a shared response to landscape history ${ }^{9}$.

An alternative hypothesis is that the principal effect of Andean mountain building in the Neogene on speciation was the formation of a geographically structured landscape matrix upon which subsequent diversification occurred. Within the humid lowland forests of the Neotropics the landscape contains mountains and rivers that restrict the movement of individuals across them (we use the term dispersal for these movements). Under this model, lineages with a longer occupation of the landscape have a higher likelihood of dispersing across geographical barriers and diversifying. In addition, lineages with lower dispersal ability are expected to accrue genetic differentiation between populations at a relatively higher rate than more dispersive lineages, leading to a higher rate of speciation ${ }^{7}$. In this model, lineage-specific attributes are predicted to be the primary determinants of species diversity within lineages ${ }^{11}$.

These two models of diversification in the Neotropics have been difficult to evaluate empirically because: (1) large-scale comparative data are needed from multiple co-distributed lineages; (2) each lineage needs to be sampled densely across its range to identify phylogeographic breaks and to estimate within-lineage species diversity; (3) the sampled lineages must encompass a range of quantifiable dispersal abilities and ecological guilds in order to test how these variables affect speciation; and (4) the phylogenetic position of each lineage must be known to approximate lineage age. We assessed the relative support for these two models in explaining standing species-level variation by characterizing recent large-scale diversification using a comparative phylogeography data set containing over 2,500 individuals from 27 widespread bird lineages in the speciesrich Neotropics (Supplementary Table 17 and Figs 1 and 2). Biological species often represent an inaccurate estimate of the true diversity in avian rainforest communities because the alpha taxonomies of most groups still require formal revision using modern methods. To minimize biases associated with species limits based on current taxonomy, we defined each lineage as all populations of a given taxon that represent, on the basis of available evidence, a monophyletic group, regardless of whether the lineage is currently treated as a single species or as a species complex that includes several closely related species. By examining relatively recent diversification at the phylogeographic scale, where extinction is less likely to have occurred, we minimized the confounding effects of extinction. Extinction is difficult to account for analytically and typically increases with time ${ }^{12}$.

The Andes, the Isthmus of Panama and large rivers of the Amazon Basin (the Amazon, Madeira and Negro rivers) are prominent features of the Neotropical landscape that interrupt the distributions of the 27 focal lineages to varying degrees (Fig. 1 and Supplementary Figs 1-27). The effect of the landscape on diversification is evident taxonomically, with distinct taxa usually located on opposite banks of Amazonian rivers, the Isthmus of Panama and the Andes. Biogeographers often treat regions delimited by these dispersal barriers as areas of endemism because of the accumulation within them of distinct taxa having common distributional ranges (Extended Data Fig. 1). The exact time of origin of the dispersal barriers separating these areas is debated ${ }^{4,5,13-16}$, but most data

\footnotetext{
${ }^{1}$ Museum of Natural Science, Louisiana State University, Baton Rouge, Louisiana 70803, USA. ${ }^{2}$ Department of Ornithology, American Museum of Natural History, New York, New York 10024 , USA. ${ }^{3}$ Department of Biological Sciences, Louisiana State University, Baton Rouge, Louisiana 70803, USA. ${ }^{4}$ Biology Department, City College of New York, New York, New York 10031, USA. ${ }^{5}$ Division of

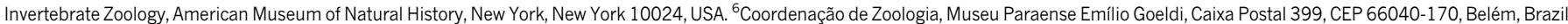

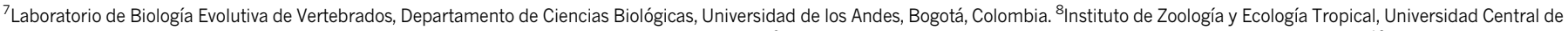

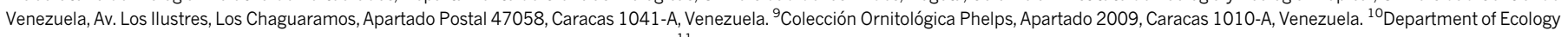
and Evolutionary Biology, University of California, Los Angeles, California 90095, USA. ${ }^{11}$ Department of Environmental Health Science, University of Georgia, Athens, Georgia 30602, USA. †Present

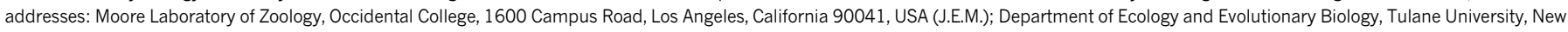

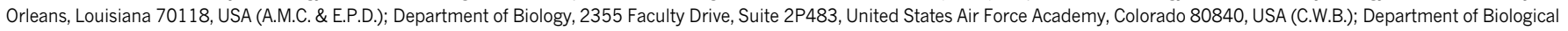
Sciences, Louisiana State University, Baton Rouge, Louisiana 70803, USA (B.C.F.).
}

*These authors contributed equally to this work. 


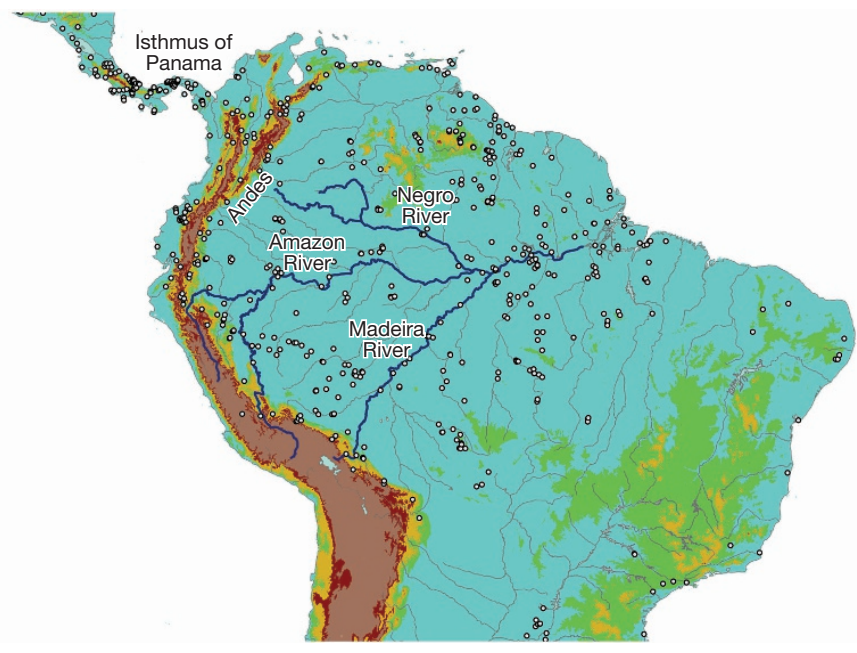

Figure $1 \mid$ Sampling within the landscape matrix. Sampling points of the 27 bird lineages (circles) and prominent dispersal barriers within the landscape matrix, including the Andes (and associated arid habitats in the Caribbean lowlands of South America), the Isthmus of Panama and three major rivers in the Amazon Basin (Amazon, Negro and Madeira Rivers).

indicate that they achieved their modern configuration during the Neogene (23-2.6 million years (Myr) ago $)^{4}$. Subsequent landscape changes during the Quaternary period (2.6 Myr ago to present) were marked by fluctuations in forest cover driven by glacial-interglacial cycles ${ }^{4,17}$, but Amazonia remained forested even during the cooler and drier glacial periods ${ }^{18}$.

Genealogies of the 27 lineages exhibited substantial variation in the timing and spatial sequence of diversification associated with barriers (Fig. 3a, Supplementary Figs 1-27 and Supplementary Table 17). To test whether divergence events across the major dispersal barriers structuring these genealogies were consistent with a single episode of vicariance associated with barrier formation we used hierarchical approximate Bayesian computation $(\mathrm{hABC})^{19}$, which is able to account for differences in genetic

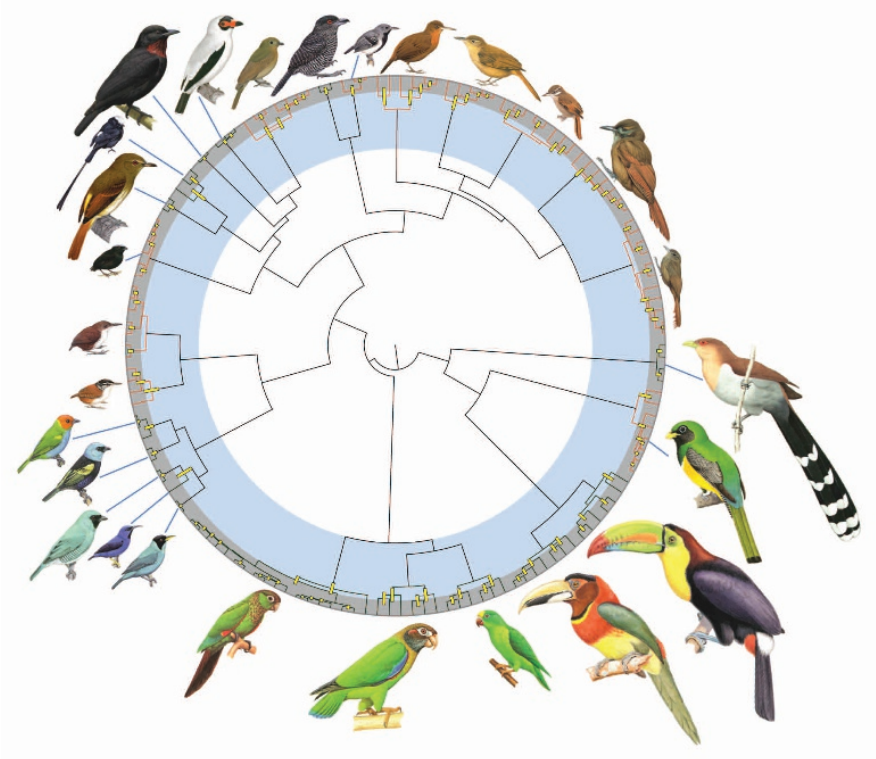

Figure $2 \mid$ Gene tree composed of 27 lineages of Neotropical birds, with species at tips inferred using a Bayesian coalescent model. An exemplar taxon for each lineage is illustrated ${ }^{30}$. Yellow bars correspond to the $95 \%$ highest posterior density for divergence times of each species. The Quaternary (2.6 Myr ago-present) and the Neogene (23-2.6 Myr ago) periods are shaded in grey and light blue, respectively. Mean stem ages for 25 of the lineages occurred within the Neogene and for two lineages within the Quaternary. Outgroups for each lineage are not included in the depicted phylogeny. drift among the 27 lineages (Extended Data Fig. 2 and Supplementary Tables 3-7). Instead of supporting a single event, the genetic data were consistent with 9 to 29 divergence events across the Andes, with each event occurring at a different time (Bayes factor $(\mathrm{Bf})=0$ when comparing $\sigma^{2} / \bar{\tau} \leq 0.01$ and $\sigma^{2} / \bar{\tau}>0.01$; Extended Data Fig. 2 and Supplementary Information). The timing $(\tau)$ of most of these divergence events was in the Pleistocene. These results suggest the Andean uplift did not have a direct cross-lineage effect on biological diversification via vicariance, but rather had an indirect role in divergence by acting as a semi-permeable barrier to post-uplift dispersal. We corroborated the above result of asynchronous cross-Andes divergences $(\mathrm{Bf}=0.13)$ using hABC analyses on multi-locus data sets (that is, $>100$ loci) generated from target capture and next-generation sequencing on a selected sample of lineages, indicating the pattern was robust to possible bias associated with inferring population history from single-locus data (Extended Data Fig. 3 and Supplementary Information). The numbers of temporally spaced events also did not support synchronous divergence across the Isthmus of Panama and the Amazonian rivers (Isthmus: 1-7 divergence events, $\mathrm{Bf}=0.00$; Amazon River: $1-3$ divergence events, $\mathrm{Bf}=0.01$; Negro River: 8-17 divergence events, $\mathrm{Bf}=0.63$; Madeira River: $3-8$ divergence events, $\mathrm{Bf}=0.66$; Extended Data Fig. 2 and Supplementary Information), a pattern consistent with the permeability of these barriers ${ }^{20}$.

We next examined to what extent speciation was influenced by the histories and ecologies of the 27 lineages. We selected two historical and two ecological summary variables previously implicated in avian diversification: (1) lineage age (a measure of evolutionary persistence), which we measured as the timing of a lineage's divergence from its sister taxon (stem age); (2) ancestral area of a lineage's origin (east or west of the Andes); (3) foraging stratum, a measure of dispersal ability linked to the behaviour of birds (canopy, high dispersal ability or understorey, low dispersal ability); and (4) niche breadth (an indirect measure of dispersal ability based on habitat preference), estimated from climate-based ecological niche models (Supplementary Information). We then used phylogenetic generalized least-squares analyses to test the effects of these variables on the number of species within each of the 27 lineages, as defined by a coalescent-based Bayesian species-delimitation method (Supplementary Information and Extended Data Fig. 4).

We found that a lineage's intrinsic ability to persist in the landscape was an important driver of speciation. The number of species within a lineage was strongly predicted by lineage age $(\triangle \mathrm{AICc}=6.9586$, where $\triangle \mathrm{AIC} c$ refers to the change in the sample size-corrected Akaike information criterion when a predictor variable was removed from the model containing all predictor variables; Fig. $3 \mathrm{~b}$, Table 1 and Supplementary Tables 12 and 16). This relationship is consistent with the idea that the longer a lineage occupies the landscape the more opportunities it has to disperse and differentiate across geographical barriers. Although a sequence of vicariant events acting on a set of co-distributed lineages could produce a similar association between lineage age and species diversity, most of the species diversity we identified originated during the Pleistocene epoch (Fig. 2 and Supplementary Table 17; $n=142 ; 75 \%$ of species $\leq 2.6 \mathrm{Myr}$ ago), after the Neogene formation of the landscape matrix, but before the Last Glacial Maximum (26,500-19,000 years ago). At deeper phylogenetic timescales, a positive association between divergence levels and lineage age has been used to explain greater species richness in areas having had more time to accumulate species ${ }^{21}$. It remains an open question whether the phylogeographic-scale processes we documented scale up to shape large-scale biodiversity patterns. To put our results into a broader temporal and spatial context would require a comparison of recent diversification events between temperate and tropical lineages ${ }^{22}$.

Ecologically, we found that foraging stratum had a significant effect on species diversity $(\triangle \mathrm{AICc}=4.0122$; Fig. 3 , Table 1 and Supplementary Tables 12 and 16), with the more dispersal-limited lineages restricted to the forest understorey exhibiting significantly higher species diversity than the more dispersive canopy lineages. This result corroborates previous work that documented the greater dispersal ability of canopy 

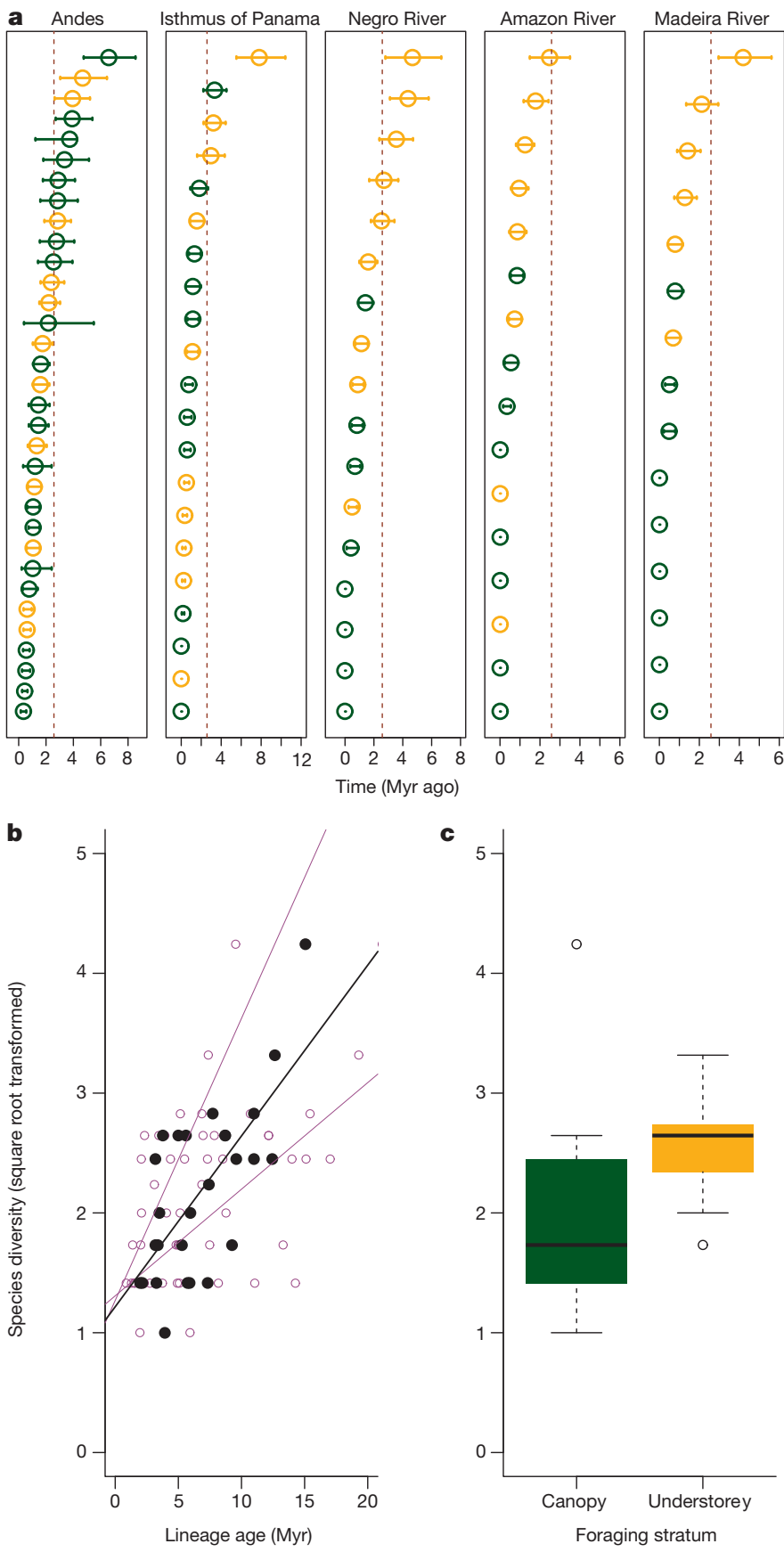

species, presumably due to the physiognomy of the canopy and the patchier distribution of food resources within $\mathrm{it}^{23,24}$. The ability of individuals to move through the landscape matrix has long-term consequences for the accumulation of diversity within lineages, assuming the lineage persists over evolutionary timescales.

Studies of biological diversification have sought a general mechanism to explain the origins of the extraordinary diversity in Amazonia ${ }^{2,4,25}$, with most concluding that landscape change by geological, climatic or marine forces is the principal driver of speciation. Using a comparative phylogeographic approach and incorporating the variability in ecology and evolutionary history among co-distributed lineages, we found that genetic patterns in birds are not easily reconcilable with a model in which diversification is a direct response to landscape change. Instead of finding the predicted shared response among lineages, our comparative analysis, and phylogeographic studies of other Amazonian organisms ${ }^{26}$, found extensive spatial and temporal discordance in genetic differentiation to
Figure $3 \mid$ Asynchronous divergence times across barriers and the influence of lineage-specific traits on species diversity. a, The variation in divergence times across barriers cannot be attributed to ecologically mediated vicariance. There was no significant association between dispersal ability and divergence times across the Andes and the Isthmus of Panama. Only part of the variance in divergence times across rivers was attributable to dispersal ability. Divergence levels across Amazonian rivers were generally shallower in canopy birds, but understorey birds diverged multiple times across each river. Circles represent mean estimates and bars represent the 95\% highest posterior density. Colour coding of the points corresponds to the foraging stratum of each lineage: understorey, orange; canopy, green. Vertical hashed lines at 2.58 million years represent the transition between the Neogene (to the right of line) and Quaternary (to the left of line). $\mathbf{b}$, Within-lineage species diversity increases with lineage (stem) age. Solid lines represent the fit of the data to a model using phylogenetic generalized least-squares analyses. Black points and line correspond to mean stem ages, and the purple points and lines correspond to the high and low values of the stem age $95 \%$ highest posterior density. c, Box plot illustrating that species diversity is significantly higher in the understorey lineages than in forest canopy lineages. The box plot shows the first, second and third quartiles, the lines are the $95 \%$ confidence intervals and the circles represent outliers. Significant associations in panels $\mathbf{a}, \mathbf{b}$ and $\mathbf{c}$ are supported by phylogenetic generalized least-squares analyses shown in Table 1 and Supplementary Tables 9-15. Statistical tests were performed independently on each data set except for divergences across rivers; all rivers were combined into a single analysis.

be the norm. For example, divergence levels across the Andes were consistent with 9 to 29 distinct divergence events (Extended Data Fig. 2). Although highly suggestive of multiple dispersal events, this variation could be explained by a single vicariant event associated with the Andean uplift if the dispersal restrictions imposed by the barrier were heavily dependent on dispersal ability, such as was reported for a taxonomically diverse group of marine organisms isolated by the formation of the Isthmus of Panama ${ }^{27}$. In a similar fashion, the emerging Andes could have first become a barrier for bird lineages with low dispersal abilities, with fragmentation of the distributions of more dispersive lineages occurring later. However, we detected no significant associations between dispersal abilities and divergence times across the Andes and the Isthmus of Panama that would support a model of ecologically mediated vicariance for these barriers (Fig. 3a and Supplementary Tables 13 and 14). For the Amazonian rivers, only part of the variance in divergence levels was explained by dispersal ability (Supplementary Table 15) because there were multiple independent divergence events within the understorey lineages (Fig. 3a and Extended Data Fig. 2). Thus, the wide range of divergences across rivers cannot be reconciled with a model of ecologically mediated vicariance. As the stem ages of 25 of the 27 lineages we examined date to the Neogene, we do not reject the possibility that the initial geographical isolation of populations at deeper phylogenetic scales was due to vicariance associated with the Andean orogeny or with the emergence of other landscape features.

The accumulation of bird species in the Neotropical landscape occurred through a repeated process of geographical isolation, speciation and expansion, with the amount of species diversity within lineages influenced by how long the lineage has persisted in the landscape and its ability to disperse through the landscape matrix. A growing body of phylogenetic

Table 1 | Phylogenetic generalized least-squares regression showing the effects of historical and ecological variables on species diversity

\begin{tabular}{lrcccc}
\hline Effect & Estimate & Standard error & \multicolumn{1}{c}{$t$ value } & $P$ & \multicolumn{1}{c}{$\Delta$ AICc } \\
\hline Lineage age & 0.1187 & 0.0283 & 4.1907 & 0.0004 & 6.9586 \\
Foraging & 0.5188 & 0.2025 & 2.5623 & 0.0178 & 4.0122 \\
stratum & & & & & \\
Ancestral origin & -0.1921 & 0.2023 & -0.9495 & 0.3527 & -1.9546 \\
Niche breadth & 1.0097 & 1.0658 & 0.9473 & 0.3538 & -1.9595
\end{tabular}

Output is from the full model and $\triangle \mathrm{AICc}$ refers to the change in AICc when each predictor variable was removed from the full model. Species diversity was square root transformed and (stem) lineage age is in units of millions of years. Full model AICc $=43.7365$; adjusted $R^{2}=0.567 ; f_{(\text {d.f.) }}=9.52_{(4,22)} ; P<0.001$; $n=27$ lineages. Model output for foraging stratum and ancestral origin corresponds to the comparison of the reference level (foraging stratum, understorey; ancestral origin, east of the Andes) for each categorical variable. 
evidence indicates that average rates of avian diversification have been relatively constant in the Neotropics ${ }^{28,29}$ and, consistent with this, our results show that tumultuous changes to the South American landscape may not have led to marked pulses in speciation. Correlations between lineage ages and the Andean uplift or Quaternary climatic events reported elsewhere ${ }^{9,25}$ are suggestive of landscape and environmental change being a component of the diversification process, but the details of how, when and to what extent these changes drove the origin of standing specieslevel diversity remain unclear. Our phylogeographic-scale analysis indicated most species-level variation postdates the Andean uplift, and our results contribute to a growing number of studies reporting dispersal events as the primary initiators of geographical isolation and speciation ${ }^{6}$. Our results also have an important conservation implication. Anthropogenic alterations of the landscape matrix by deforestation and climate change affect not only the evolutionary persistence of rainforest lineages, but also the occurrence of cross-barrier dispersal events within lineages that lead to new biological diversity.

Online Content Methods, along with any additional Extended Data display items and Source Data, are available in the online version of the paper; references unique to these sections appear only in the online paper.

Received 6 April; accepted 17 July 2014.

Published online 10 September; corrected online 19 November 2014 (see full-text HTML version for details).

1. Nelson, G. J. \& Platnick, N. I. Systematics and Biogeography: Cladistics and Vicariance Vol. 214 (Columbia Univ. Press, 1981).

2. Haffer, J. Speciation in Amazonian forest birds. Science 165, 131-137 (1969).

3. Mayr, E. Systematics and the Origin of Species, from the Viewpoint of a Zoologist No. 13 (Harvard Univ. Press, 1942).

4. Hoorn, C. F. P. et al. Amazonia through time: Andean uplift, climate change, landscape evolution and biodiversity. Science 330, 927-931 (2010).

5. Ribas, C. C., Aleixo, A., Nogueira, A. C., Miyaki, C. Y. \& Cracraft, J. A palaeobiogeographic model for biotic diversification within Amazonia over the past three million years. Proc. R. Soc. Lond. B 279, 681-689 (2012).

6. Sanmartín, I., van der Mark, P. \& Ronquist, F. Inferring dispersal: a Bayesian approach to phylogeny-based island biogeography, with special reference to the Canary Islands. J. Biogeogr. 35, 428-449 (2008).

7. Wakeley, J. \& Aliacar, N. Gene genealogies in a metapopulation. Genetics 159, 893-905 (2001).

8. Udvardy, M. D. F. \& Papp, C. S. Dynamic Zoogeography (Van Nostrand Reihold Company, 1969).

9. Antonelli, A. et al. in Amazonia, Landscape and Species Evolution (eds Hoorn, C. \& Wesselingh, F.P.) 386-404 (Blackwell, 2010).

10. Chapman, F. M. The Distribution of Bird-Life in Colombia: a Contribution to a Biological Survey of South America Vol. 36 (American Museum of Natural History, 1917).

11. Burney, C. W. \& Brumfield, R. T. Ecology predicts levels of genetic differentiation in neotropical birds. Am. Nat. 174, 358-368 (2009).

12. Rabosky, D. L. Extinction rates should not be estimated from molecular phylogenies. Evolution 64, 1816-1824 (2010).

13. Gregory-Wodzicki, K. M. Uplift history of the Central and Northern Andes: a review. Geol. Soc. Am. Bull. 112, 1091-1105 (2000).

14. Campbell, K. E. Jr, Frailey, C. D. \& Romero-Pittman, L. The Pan-Amazonian Ucayali Peneplain, late Neogene sedimentation in Amazonia, and the birth of the modern Amazon river system. Palaeogeogr. Palaeoclimatol. Palaeoecol. 239, 166-219 (2006)

15. Latrubesse, E. M. et al. The late Miocene paleogeography of the Amazon Basin and the evolution of the Amazon River system. Earth Sci. Rev. 99, 99-124 (2010).

16. Montes, C. et al. Evidence for middle Eocene and younger land emergence in central Panama: implications for isthmus closure. Geol. Soc. Am. Bull. 124, 780-799 (2012).

17. Cheng, H. et al. Climate change patterns in Amazonia and biodiversity. Nature Commun. 4, 1411 (2013).
18. Bush, M. B., Gosling, W. D. \& Colinvaux, P. A. in Tropical Rainforest Responses to Climatic Change Ch. 3, 61-84 (Springer Praxis Books, 2011).

19. Hickerson, M. J., Stahl, E. A. \& Takebayashi, N. msBayes: pipeline for testing comparative phylogeographic histories using hierarchical approximate Bayesian computation. BMC Bioinform. 8, 268 (2007).

20. Naka, L. N. N. et al. The role of physical dispersal barriers in the location of avian suture zones in the Guiana Shield, northern Amazonia. Am. Nat. 179, E115-E132 (2012).

21. Wiens, J. J. The causes of species richness patterns across space, time, and clades and the role of "ecological limits". Q. Rev. Biol. 86, 75-96 (2011).

22. Weir, J. T. \& Schluter, D. The latitudinal gradient in recent speciation and extinction rates of birds and mammals. Science 315, 1574-1576 (2007).

23. Greenberg, R. The abundance and seasonality of forest canopy birds on Barro-Colorado Island, Panama. Biotropica 13, 241-251 (1981).

24. Loiselle, B. A. Bird abundance and seasonality in a Costa Rican lowland forest canopy. Condor 90, 761-772 (1988).

25. Rull, V. Neotropical biodiversity: timing and potential drivers. Trends Ecol. Evol. 26, 508-513 (2011).

26. Turchetto-Zolet, A. C., Pinheiro, F., Salgueiro, F. \& Palma-Silva, C. Phylogeographical patterns shed light on evolutionary process in South America. Mol. Ecol. 22, 1193-1213 (2013).

27. Lessios, H. A. The great American schism: divergence of marine organisms after the rise of the Central American isthmus. Annu. Rev. Ecol. Evol. Syst. 39, 63-91 (2008).

28. Jetz, W., Thomas, G. H., Joy, J. B., Hartmann, K. \& Mooers, A. O. The global diversity of birds in space and time Nature 491, 444-448 (2012)

29. Derryberry, E. P. et al. Lineage diversification and morphological evolution in a large-scale continental radiation: the Neotropical ovenbirds and woodcreepers (Aves: Furnariidae). Evolution 65, 2973-2986 (2011)

30. del Hoyo, J., Elliott, A., Sargatal, J. \& Christie D. A. (eds) Handbook of the Birds of the World (Lynx Edicions, 1992-2013).

Supplementary Information is available in the online version of the paper.

Acknowledgements We thank the collectors, preparators, collection managers and curators of vouchered tissue samples who made this study possible. We thank the following people and institutions for providing samples: D. Dittmann, F. Sheldon (LSUMZ), N. Rice (ANSP), M. Robbins (KU), D. Willard, S. Hackett (FMNH), G. Graves, J. Dean (USNM), J. Cracraft, P. Sweet, T. Trombone (AMNH), S. Birks, J. Klicka (UWBM), K. Bostwick, I. Lovette (CUMV), B. Hernández-Baños, A. Navarro (MZFC), D. López (IAvH-BT), F. G. Stiles (ICN), M. Lentino (COP), F. Raposo, C. Miyaki (LGEMA, USP) and Museo de Historia Natural de la Universidad de los Andes. This study was supported by NSF awards to R.T.B. (DEB-0841729), M.J.H. (DEB 1253710; DEB 1343578) and CUNY HPCC (CNS-0855217), the Coypu Foundation, Brazilian Research Council (Conselho Nacional de Desenvolvimento Científico e Tecnológico) (grant numbers: 574008-2008-0; 490131/2009-3; 310593/2009-3; 574008/2008-0; 563236/ 2010-8 and 471342/ 2011-4) and FAPESPA awards (ICAAF 023/2011) to A.A., and support from CDCH and INPMA to J.P.-E. We thank G. Thomas, N. Gutierrez-Pinto, N. Reid, G. Bravo, J. Miranda, G. Seeholzer, C. Salisbury, C. Cooney, R. Bryson Jr, B. Riddle, N. Takebayashi, B. Winger, V. Chua and J. Weckstein for their assistance, comments and feedback. We thank Lynx Edicions and E. Badia for granting us permission to reuse bird plates from the Handbook of Birds of the World in Fig. 2.

Author Contributions B.T.S. performed ecological niche modelling and conducted all statistical analyses except for $\mathrm{hABC}$ analyses, which were performed and interpreted by M.J.H. and X.X. J.E.M., A.M.C., A.A., C.D.C., J.P.-E., C.W.B., E.P.D., J.P. and S.F. assisted with sampling and mitochondrial data collection. B.C.F., M.G.H., T.C.G. and B.T.S. collected ultraconserved element multi-locus sequence capture data. R.T.B. conceived the study. R.T.B., C.D.C., A.A., J.P.-E., B.T.S. and J.E.M. designed the study. B.T.S. and R.T.B. wrote the paper with help from M.J.H., M.G.H., C.D.C., J.E.M., A.M.C., A.A., J.P.-E., B.C.F. and T.C.G.

Author Information Mitochondrial sequences generated for this study were deposited at GenBank under accession numbers KM079656-KM081611. This work was conducted under Louisiana State University Institutional Animal Care and Use Committee Protocol 09-001. Reprints and permissions information is available at www.nature.com/reprints. The authors declare no competing financial interests. Readers are welcome to comment on the online version of the paper. Correspondence and requests for materials should be addressed to R.T.B. (robb@lsu.edu). 


\section{RESEARCH LETTER}

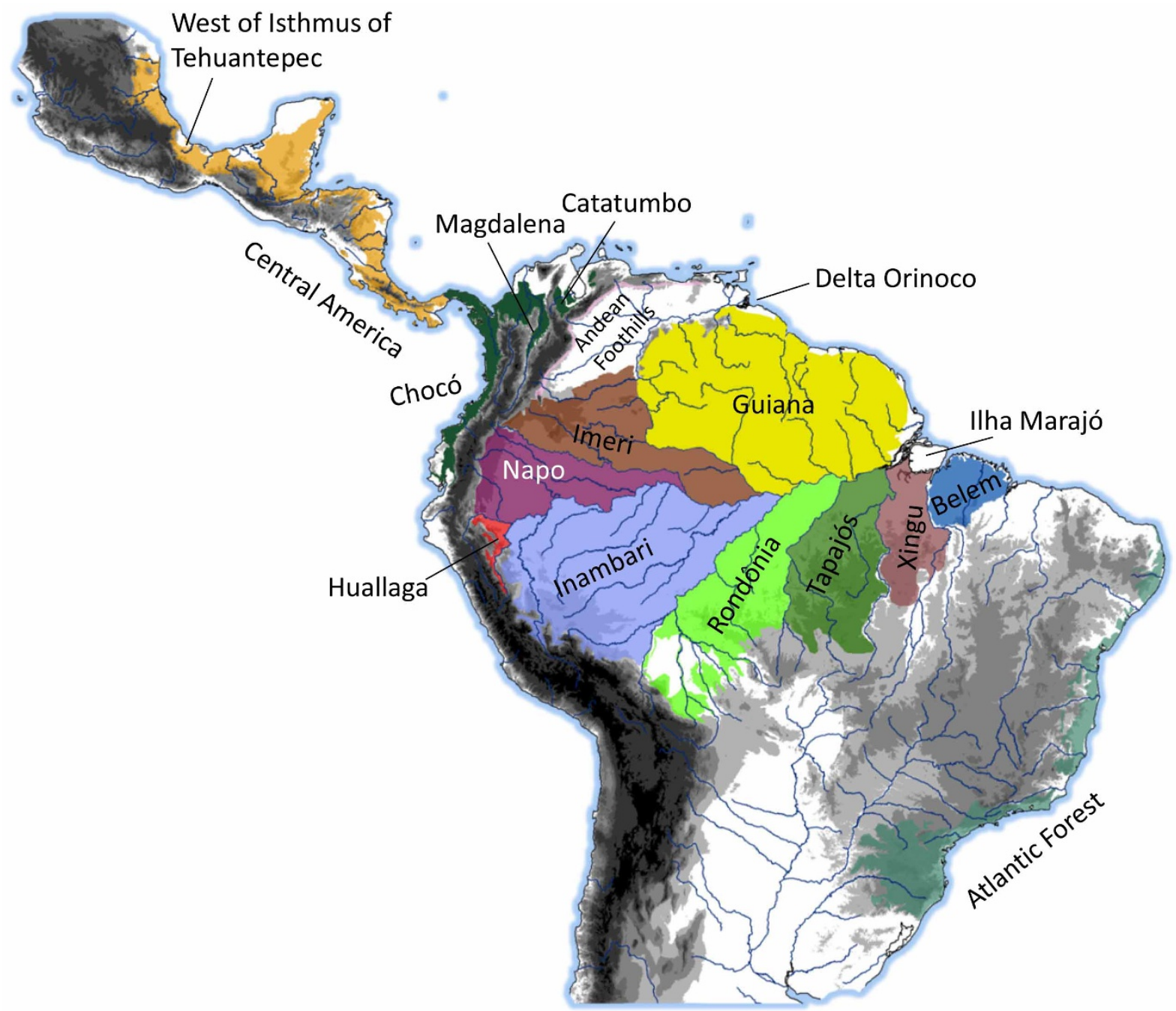

Extended Data Figure $1 \mid$ Areas of endemism for lowland rainforest birds in Central and South America. A full description of the geographical limits of each area is available in the Supplementary Information. 

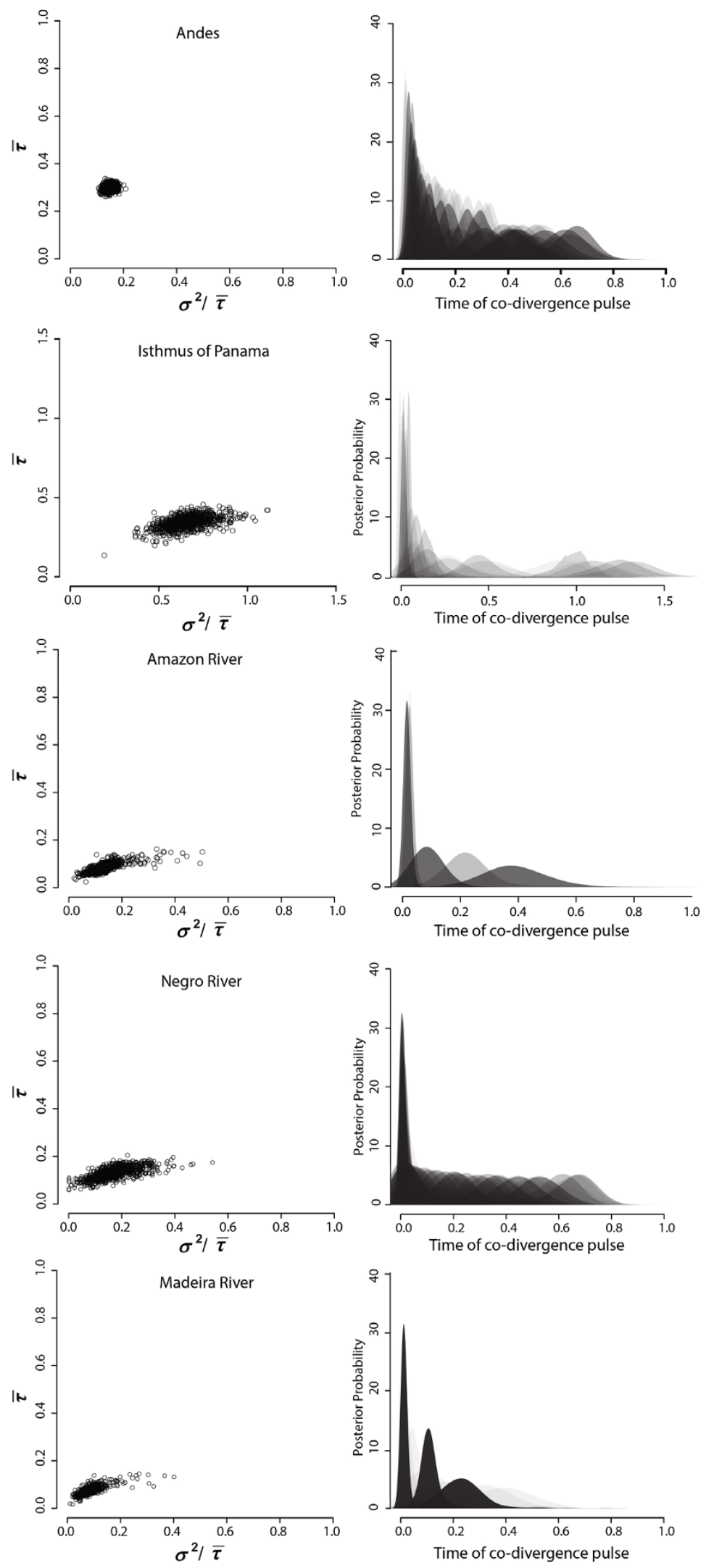

Extended Data Figure $2 \mid$ hABC output showing estimates of mean and dispersion indices of population divergence times and times of co-divergence pulses inferred from mitochondrial DNA. The left panels illustrate the approximate joint posterior estimates of $\sigma^{2} / \bar{\tau}$, the dispersion index of $\tau$ and $\bar{\tau}$, the mean of $\tau$ across $n$ population pairs, where $\tau_{i}$ is the divergence time of the $i$ th of $n$ population-pairs and $\bar{\tau}$ is scaled in coalescent time units of $4 \bar{N}$ generations where $\bar{N}$ is the mean effective population size averaged across

population-pairs. The right panels depict the posterior distributions of the relative times of the co-divergence pulses across barriers, scaled by coalescent units. The shading intensity of each distribution is conditional on the posterior probability of $\psi$, the associated number of different pulses of co-divergence across each barrier. Sample sizes for each barrier: Andes, $n=29$; Isthmus of Panama, $n=14$; Amazon River, $n=14$; Negro River, $n=17$; Madeira River, $n=14$. 


\section{RESEARCH LETTER}

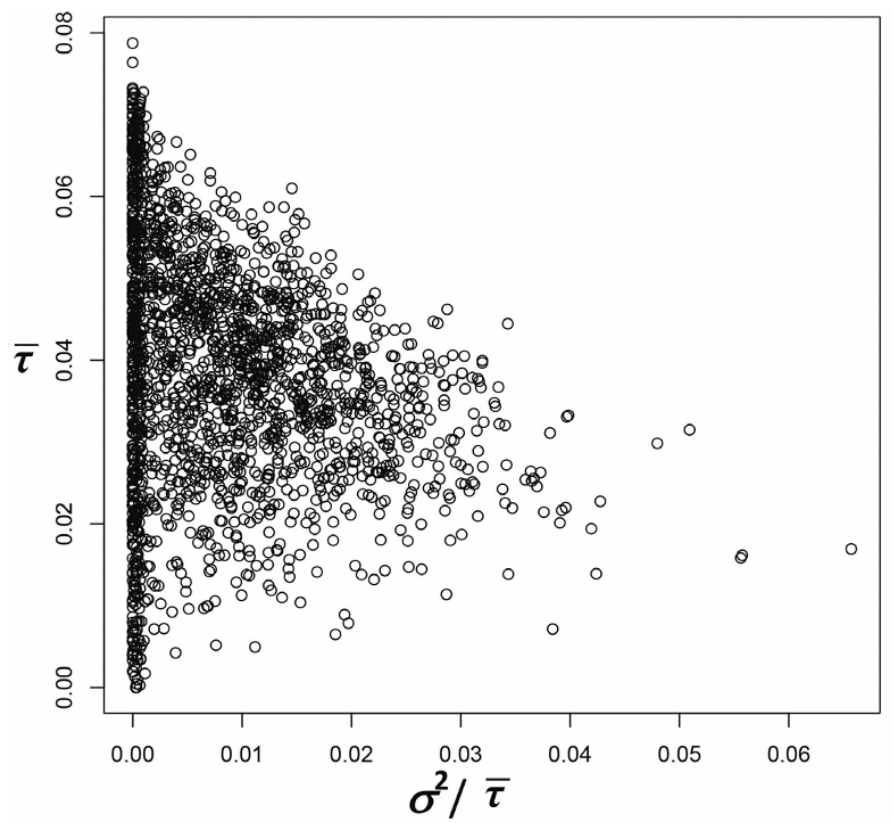

Extended Data Figure $3 \mid$ hABC output showing estimates of mean and dispersion indices of population divergence times across the Andes inferred from ultraconserved elements (UCEs). Left panel illustrates the approximate joint posterior estimates of $\sigma^{2} / \bar{\tau}$, the dispersion index of $\tau$ and $\bar{\tau}$, the mean of $\tau$ across $n$ population-pairs, where $\tau_{i}$ is the divergence time of the $i$ th of $n$

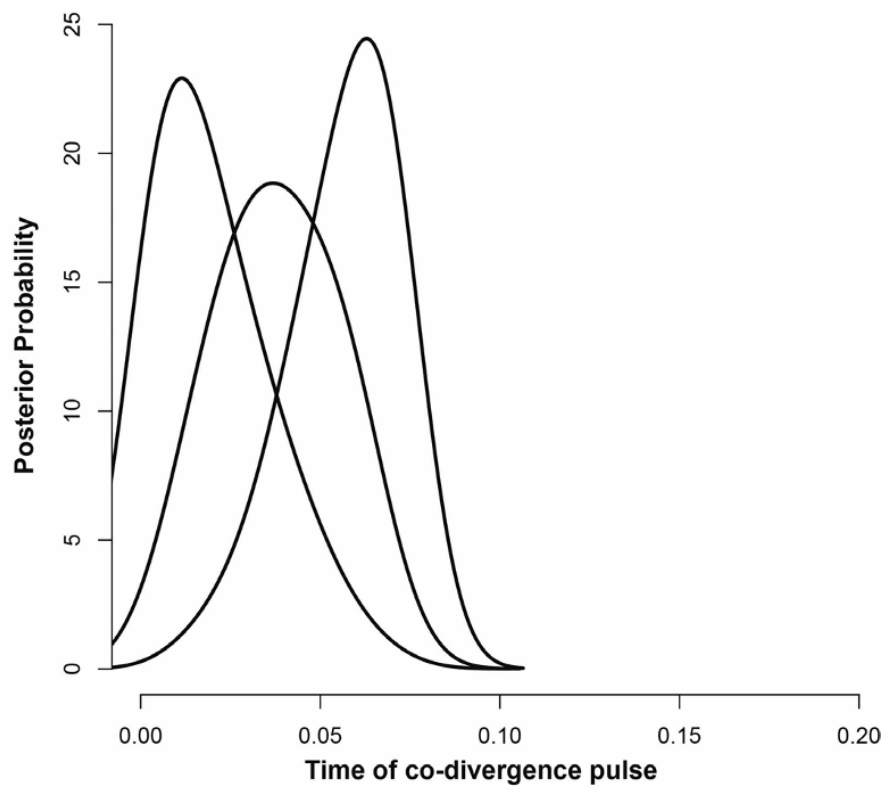

population-pairs and $\bar{\tau}$ is scaled in coalescent time units of $4 \bar{N}$ generations where $\bar{N}$ is the mean effective population size averaged across population-pairs. The right panel depicts the posterior distribution of the relative times of the codivergence pulses across the Andes $(n=5)$ scaled by coalescent units. 


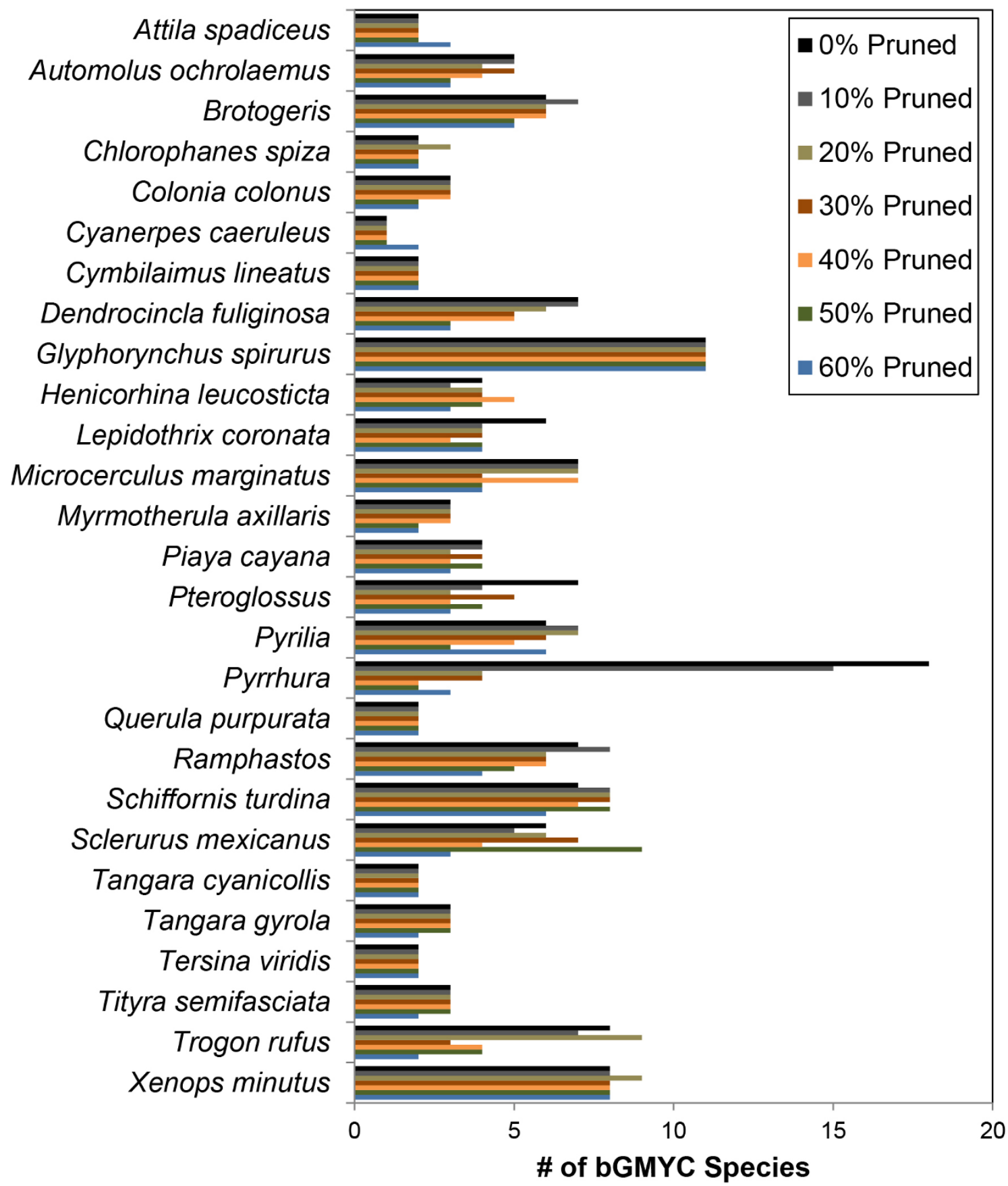

Extended Data Figure 4 $\mid$ Bar plot showing the number of estimated species using a Bayesian general mixed Yule-coalescent (bGMYC) model from

lineage correspond to the percentage $(0-60 \%)$ of individuals randomly pruned complete and randomly pruned data sets. The coloured columns for each 